\title{
Surface Josephson plasma waves in a high-temperature
} superconductor

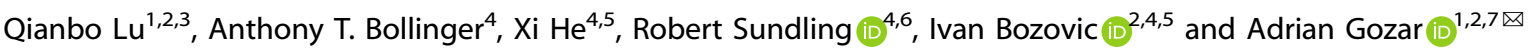

Electron density oscillations with acoustic dispersions and sustained at boundaries between different media provide information about surface and interface properties of heterostructures. In ultrathin metallic films these plasmonic excitations are heavily damped. Superconductivity is predicted to reduce dissipation allowing detection of these resonances. Emerging low-loss interface Cooper-pair waves have been studied before, however, the observation of surface-confined Josephson plasmons in highly anisotropic superconductors has remained elusive. Here, we report on generation and coupling to these excitations in an ultrathin single-crystal film of high-temperature superconductor $\mathrm{La}_{1.85} \mathrm{Sr}_{0.15} \mathrm{CuO}_{4}$. The film becomes brighter than Au below the critical temperature when probed with sub-gap THz photons. We show that the enhanced signal in the superconducting state, which can be visualized with a spatial resolution better than $\lambda / 3000$, originates from near-field coupling of light to surface Josephson plasmons. Our results open a path towards non-invasive investigation of enhanced superconductivity in artificial multilayers, buried interface states in topological heterostructures, and non-linear phenomena in Josephson devices.

npj Quantum Materials (2020)5:69; https://doi.org/10.1038/s41535-020-00272-8

\section{INTRODUCTION}

Propagating sound-like collective modes in superfluids are converted to higher energy plasma modes in superconductors ${ }^{1}$. Several decades ago, low-lying excitations with linear dispersions were detected however in the microwave region, in aluminum films ${ }^{2}$. These are known as Carlson-Goldman modes. They consist of balanced oscillations of supercurrents and normal carriers ${ }^{3}$ and are overdamped except in a narrow region close to the superconducting temperature. In thin films and superlattices, a second type of superfluid acoustic mode should exist, where the electromagnetic field is confined to the interfaces between superconducting and dielectric regions ${ }^{4}$. Two main obstacles make the observation of these excitations difficult for traditional, far-field, optical techniques ${ }^{5-7}$. One is sensitivity, i.e. the capability to distinguish surface modes from bulk contributions. The other is generic to acoustic branches and entails overcoming the momentum mismatch between free-space photons and gapless plasmons. This can be realized in principle by coupling the evanescent waves through a prism, by periodic nano-fabricated arrays, or by bringing a nano-sized light source close to the sample ${ }^{6,8,9}$. Artificial periodic corrugation was indeed used to observe the emergence of microwave surface waves in superconductors $^{10-12}$. These studies highlighted the role of reduced dissipation in the superfluid state and established these materials as candidates for temperature-tunable plasmonic structures ${ }^{10,13}$. In this work, we use the second, scattering-based, approach, see Fig. 1, which has the benefit of increased spatial resolution due to the nanometer probe size.

There is also a practical interest in materials hosting stacks of conductive metallic sheets ordered with atomic scale precision as they can be used as building blocks for plasmonic devices. Along with doped semiconductor superlattices or modern twodimensional (2D) materials, superconducting cuprates are examples of such layered systems ${ }^{14-16}$. Coupling mechanisms and the requirement of large momentum transfers for mapping dispersion branches gave electron energy loss spectroscopy the leading role in the study of plasmons, including pioneering work which enabled the extension of this concept to surfaces and interfaces ${ }^{17}$. Potential applications are met with substantial challenges because of electrical losses in conventional metallic structures ${ }^{18}$. The same problem is present and amplified in the normal state of superconductors with a high critical temperature $\left(T_{c}\right)$. Topological protection was found to strongly suppress damping of acoustic plasmon excitations propagating on the surface of topological insulators ${ }^{19,20}$. Dissipation is also expected to be significantly reduced below $T_{\mathrm{c}}$ in superconductors ${ }^{4}$. Optical probing of these materials in the sub-gap regime are of interest because below $T_{\mathrm{c}}$ they are predicted to support low-loss plasmon waves and confinement of the photon field at deep subwavelength scales ${ }^{13}$. Near-field optics brings in the additional advantage of higher spatial resolution along with sub-surface sensitivity ${ }^{21}$. For high- $T_{c}$ cuprates, the inherent non-linearity of the Josephson coupling between the superconducting $\mathrm{CuO}_{2}$ planes may enable photonic applications exploiting light-matter interactions in the $\mathrm{THz}$ range $\mathrm{e}^{22}$, with superfluid surface plasmon excitations predicted to play a special role $7,23,24$. The main result of this work is the observation of these modes in strongly anisotropic single-crystal superconductors, where their energy and dispersions depend crucially on the Josephson coupling between adjacent layers.

Systems of coupled metallic layers harbor a variety of plasmonic branches and here we describe their salient features (see also Supplementary Note 4 and Supplementary Fig. 3). There is a very large energy cost associated with excitations of plasmons in bulk metals. If the same materials are confined to $2 D$, this energy becomes vanishingly small in the long-wavelength limit; the oscillation frequency scales as $\omega(q \rightarrow 0) \propto q^{1 / 2}$, where $q$ is the inplane momentum ${ }^{25}$. Quasi-2D surface excitations confined to planar interfaces between media with dielectric permittivities of

\footnotetext{
${ }^{1}$ Department of Applied Physics, Yale University, New Haven, CT 06520, USA. ${ }^{2}$ Energy Sciences Institute, Yale University, West Haven, CT 06516, USA. ${ }^{3}$ Shaanxi Institute of Flexible Electronics, Northwestern Polytechnical University, 710072 Xi'an, China. ${ }^{4}$ Brookhaven National Laboratory, Upton, NY 11973, USA. ${ }^{5}$ Department of Chemistry, Yale University, New Haven, CT 06520, USA. ${ }^{6}$ Zensoft, Inc., Madison, WI 53705, USA. ${ }^{7}$ Department of Physics, Yale University, New Haven, CT 06520, USA. ${ }^{\circledR}$ email: adrian.gozar@yale.edu
} 

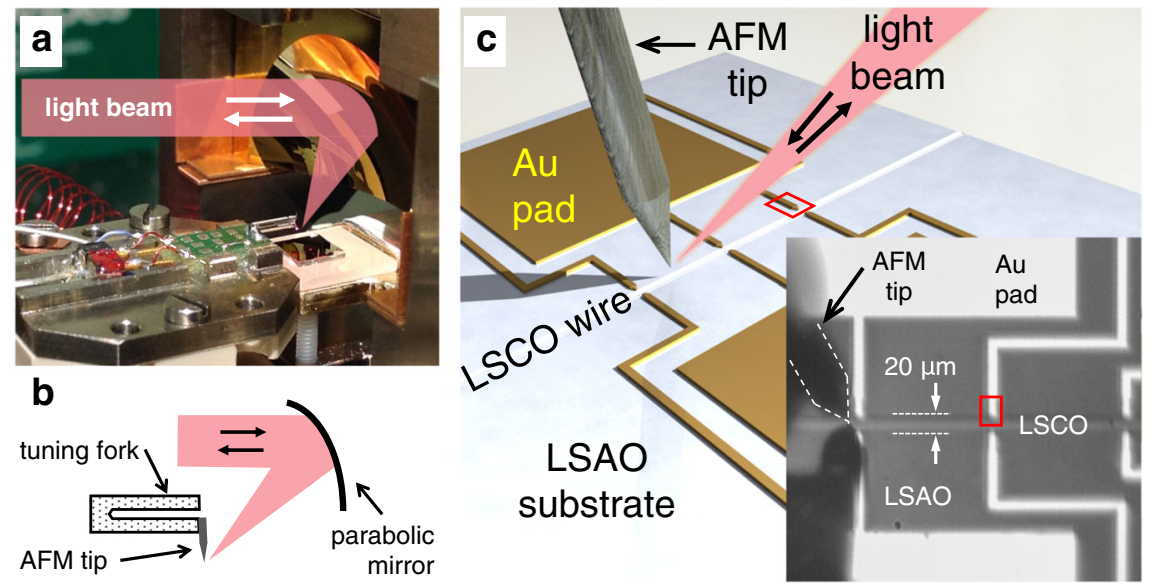

Fig. 1 The experimental system and the measured device. a A close-up of the cryogenic AFM-SNOM sample space showing the tuning fork mount, the sample holder and the path of the light beam reflecting off the parabolic mirror. The two arrows indicate light propagation directions in our back-scattering geometry. $\mathbf{b}$ Schematic showing the collimated beam being focused by the parabolic mirror onto the apex of the AFM tip. The AFM tip is an etched metallic wire glued to a prong of the quartz tuning fork sensor (see Methods). c The schematic of the measured device shows the light beam, the AFM tip, the Au pads, the $20 \mu \mathrm{m}$ wide strip of $13 \mathrm{~nm}$ thick LSCO film and the LaSrAlO ${ }_{4}$ substrate. During the measurements the tip was positioned next to lateral LSCO-Au contacts such as the one marked with a red rectangle. Similar data were obtained from all measured contact regions, attesting to the robustness of the results. The inset of this panel shows an actual CCD image of the investigated sample area. Visible on the left side of the inset is the etched AFM tip and its reflection in the substrate. The outline of the tip is emphasized by while dashed lines.

opposite sign are also gapless but they display a sound-like dispersion $\omega(q \rightarrow 0) \propto q$, see for example ref. ${ }^{9}$. Interestingly, a change from the expected square root to linear dispersion for $2 \mathrm{D}$ electronic states is also generated by bulk-crystal screening effects: examples are the acoustic surface plasmons in metals such as those in $\mathrm{Be}(0001)^{26}$. A cross-over between these two regimes can be achieved as well in a configuration where the $2 \mathrm{D}$ electron gas is screened by the proximity of a conductive planar electrode/gate ${ }^{12}$, or by stacking $2 \mathrm{D}$ conductive and insulating layers in alternation on top of one another ${ }^{27}$. The latter geometry exhibits the (quasi-)2D to 3D panoply of gapped or gapless plasmon dispersions $\omega\left(q, k_{\mathrm{z}}\right)$ as dictated by $k_{\mathrm{z}}$ the momentum perpendicular to the planes ${ }^{28}$. In addition to these propagating guided modes, superlattices also display their characteristic surface-confined acoustic modes, where the electric and magnetic fields decay exponentially with the distance both inside and outside the layered material ${ }^{6,29}$.

In high- $T_{\mathrm{c}}$ copper oxides the normal state transport is extremely anisotropic: it is metallic in the $a b$-plane (parallel to the $\mathrm{CuO}_{2}$ layers) and insulating/incoherent in the perpendicular, $c$-axis, direction. Below $T_{c}$ copper oxides behave like a series array of intrinsic Josephson junctions ${ }^{14}$. Tunneling between $\mathrm{CuO}_{2}$ planes allows propagation of bulk plasmons only above $\omega_{\mathrm{J}}$, the screened Josephson plasma energy $y^{6,8,23}$. Josephson plasma edge can be probed with far-field techniques and its measured value ranges from $\omega_{\mathrm{J}} \sim 50 \mathrm{GHz}$ in $\mathrm{Ba}_{2} \mathrm{Sr}_{2} \mathrm{CaCu}_{2} \mathrm{O}_{8}{ }^{30}$ to $\sim 1.5 \mathrm{THz}$ in $\mathrm{La}_{2-x} \mathrm{Sr}_{x} \mathrm{CuO}_{4}{ }^{31}$ and even higher in $\mathrm{YBa}_{2} \mathrm{Cu}_{4} \mathrm{O}_{8}{ }^{32}$. Surface Josephson plasma waves (SJPWs) remain the only excitations with acoustic dispersion in these materials. An anisotropic two-fluid model was shown to capture the dielectric properties of the copper oxides ${ }^{33}$ :

$\varepsilon_{a}(\omega)=\varepsilon_{\infty, a}-\frac{\omega_{\mathrm{pS}, a}^{2}}{\omega^{2}}-\frac{\omega_{\mathrm{pN}, a}^{2}}{\omega^{2}+i \Gamma \omega}, a=a b, c$.

Here " $S$ " and " $N$ " stand for the superfluid and normal components, $\omega_{\mathrm{p}}$ is the plasma frequency (for the $c$-axis direction $\left.\omega_{\mathrm{J}}=\omega_{\mathrm{pS}, \mathrm{c}} / \sqrt{ } \varepsilon_{\infty, \mathrm{c}}\right)$ and $\Gamma$ is the scattering rate. The first term in Eq. (1) follows from the London model and the second is a Drude-model description of the uncondensed carriers. In optimally doped $\mathrm{La}_{1.85} \mathrm{Sr}_{0.15} \mathrm{CuO}_{4}$ (LSCO) the ratio of the in-plane to the $c$-axis superfluid plasma frequencies is $\omega_{\mathrm{ps}, \mathrm{c}} / \omega_{\mathrm{ps}, \mathrm{ab}} \approx 25$ at low temperatures ${ }^{31,34}$.

\section{RESULTS}

Film synthesis and near-field data

We achieved coupling to SJPWs in a $13 \mathrm{~nm}$ thick LSCO film by using a customized cryogenic system based on atomic force microscopy combined with scanning near-field optical microscopy $^{35,36}$ (AFM-SNOM), see Fig. 1, Methods and Supplementary Note 1. For film synthesis, we have used an advanced atomiclayer-by-layer molecular beam epitaxy (ALL-MBE) system, a technique proven to provide the highest-quality, single-crystal LSCO films with atomically smooth surfaces and interfaces ${ }^{37}$. The surface morphology and crystalline structure of the film were monitored by reflection high-energy electron diffraction, in real time. A source of pure ozone was used to ensure sufficient oxidation under high-vacuum conditions. Single-crystal $\mathrm{LaSrAlO}_{4}$ substrates were polished perpendicular to the crystallographic [001] direction. The films are epitaxially locked to the substrate and pseudomorphic; the $\mathrm{CuO}_{2}$ layers are parallel to the LSCO film surface. During the growth, we kept the ozone partial pressure at $p=2 \times 10^{-5}$ Torr and substrate temperature at $T_{\mathrm{s}}=650^{\circ} \mathrm{C}$. To protect the film surface, we cover the films in situ with $10 \mathrm{~nm}$ thick layer of gold, deposited at room temperature. The device shown in the inset of Fig. 1c was patterned using photolithography and Argon-ion milling the LSCO film into a $10 \mathrm{~mm}$ long, $20 \mu \mathrm{m}$ wide strip with 64 lateral leads to large contact pads. The protective gold layer was removed from the active LSCO strip by an appropriate gold etch.

The AFM-SNOM system was optimized for laser light access and large collection throughput, as well as for AFM tip positioning and device visualization ${ }^{38}$, see Fig. 1. All data shown here were acquired with photon energies $\omega=26.7 \mathrm{~cm}^{-1}(0.8 \mathrm{THz}$ or $3.52 \mathrm{meV}$ ), corresponding to a wavelength $\lambda=375 \mu \mathrm{m}$. Gold pads contacting Hall bar devices were used as the optical reference material. AFM-SNOM scans with typical size of $1.3 \times 8 \mu \mathrm{m}$ (more than 2 orders of magnitude smaller than the photon wavelength) were taken in the temperature range $20 \mathrm{~K}<T<300 \mathrm{~K}$. These images are shown in Fig. 2a, b. The near-field signal was 
a

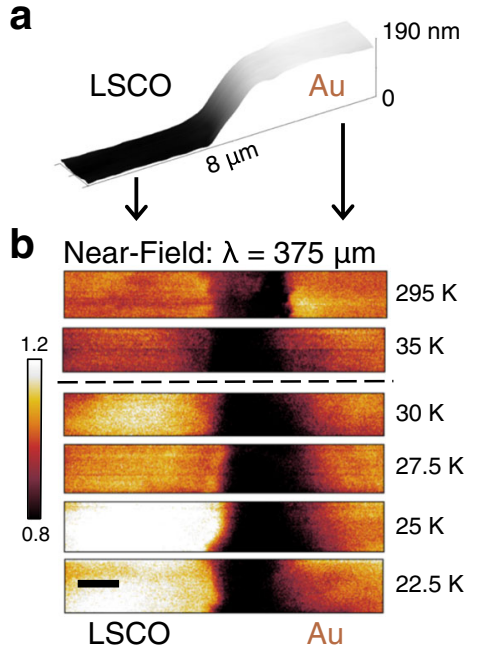

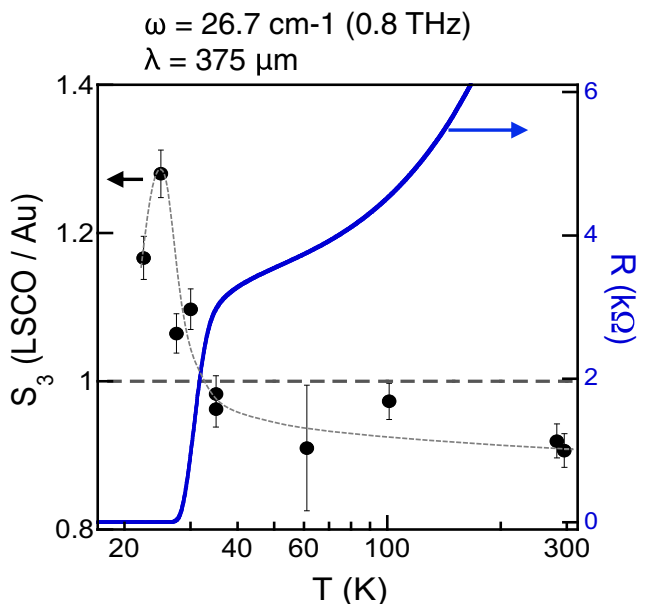

Fig. 2 Temperature dependence of the near-field signal acquired with $\omega=26.7 \mathrm{~cm}^{-1}(0.8 \mathrm{THz})$ photon energy. a 3D view of the AFM topography acquired at $T=22.5 \mathrm{~K}$ around the LSCO-Au contact area. Dark/bright areas on the left/right correspond to LSCO and Au, respectively. $\mathbf{b}$ Temperature-dependent near-field amplitude at several temperatures. LSCO and Au regions are indicated by arrows and labels at the bottom. The SNOM signal from the Au contact in each SNOM scan is normalized to unity. Dark areas between LSCO and Au are induced by the broad lithographic edge and were not used in the quantitative analysis. The scale bar in the bottom panel is $1 \mu \mathrm{m}$. c The black circles: near-field $S_{3}$ signal from LSCO normalized to Au, obtained from the data in panel (b) as a function of temperature, in log-scale. The graydashed line is a guide for the eye. The blue solid line: temperature-dependent resistance, $R(T)$, the right vertical scale.

demodulated up to the 4th harmonic of the AFM tapping frequency, see Supplementary Note 1 . We focus here on the data corresponding to the 3rd harmonic, $S_{3}$.

In Fig. $2 b$ we compare the temperature dependence of the near-field signal with that of the device resistance, $R(T)$. The corresponding temperatures are indicated to the right of each scan. LSCO regions are to the left and Au contact areas to the right in the panels. The dashed line between $T=35 \mathrm{~K}$ and $T=30 \mathrm{~K}$ scans separates the data collected at temperatures collected above and below $T_{\mathrm{c}}$. The error bars are primarily determined by surface roughness and the imperfections at the edge of the sample due to lithography. Good reproducibility in the data is demonstrated by scans acquired at the same temperatures on cooling and warming during different runs for the same contact and also at different LSCO-Au contacts. The main experimental observation is that, when probed with energies of $\omega=26.7 \mathrm{~cm}^{-1}$ $(0.8 \mathrm{THz})$, the near-field intensity ratio between LSCO and $\mathrm{Au}$ changes substantially when the HTS sample enters the superconducting state, see Fig. 2c. Above $T_{c}$ this ratio is fairly temperature independent, staying below unity around a value $\sim 0.95$. There is an abrupt rise below $T_{c}$ where the relative intensity reaches a peak value close to 1.3 at $T \approx 25 \mathrm{~K}$. The signal decreases slightly but remains above unity upon further cooling to $T=20 \mathrm{~K}$. No such changes are observed if the sample is probed with midinfrared photon energies $\omega \approx 1000 \mathrm{~cm}^{-1}$ ( $30 \mathrm{THz}$ or $125 \mathrm{meV}$ ), which are above the superconducting gap in LSCO.

\section{Theoretical modeling and interpretation}

The energy scale and the non-monotonic temperature dependence of the near-field signal across the superconducting transition provide strong constraints to possible interpretations of our data. Phonons do not display any abrupt changes at $T_{c}$. Moreover, the energies of optical and acoustic branches are either too high or too low to match the energy and range of momenta probed in our experiment ${ }^{39}$. Long wavelength and low energy magnetic excitations are already overdamped at much smaller $\mathrm{Sr}$ concentrations $^{40}$. Local antiferromagnetic fluctuations persist at optimal doping ${ }^{41}$ but they are at much higher energies, around
0.2-0.5 eV, and they also do not display any abrupt changes with temperature upon crossing $T_{\mathrm{c}}$. Increased scattering due to larger far-field reflection coefficients in the superconducting state cannot account for the observations because of their temperature dependence ${ }^{31,42}$, see also the discussion in Supplementary Note 5. We can also rule out electronic scenarios associated with both the critical behavior of the dielectric function around the percolation threshold ${ }^{43,44}$. A consideration of the energy scales, near-field coupling mechanisms, temperature dependence across the superconducting transition as well as of the parity and momentum selection rules can explain why scenarios invoking more exotic superconductivity-induced collective modes are very unlikely to account for our results ${ }^{2,45-47}$ (a more detailed discussion in connection to these aspects can be found at the end of Supplementary Note 5).

In contrast, we show here that SJPWs explain observations quite naturally. This scenario accounts for all key features of our experiment: the coupling mechanism, the abrupt and nonmonotonic behavior of the relative LSCO/Au signal with reversal of intensity upon crossing $T_{\mathrm{c}}$ as well as the energy scale which is

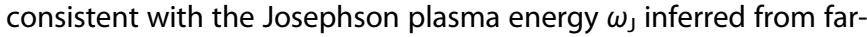
field measurements. Coupling to plasmonic excitations is enabled by evanescent modes waves with high in-plane momenta in the proximity of the AFM tip and it is well documented in the literature ${ }^{48}$. The peaks in the near-field signal are correlated to energies of surface modes. The dispersion $\omega(q)$ for the propagating electromagnetic mode confined to the planar interface between an isotropic and an optically uniaxial material is implicitly given by, see Supplementary Note 3:

$q=\frac{\omega}{c} \sqrt{\frac{\varepsilon_{1} \varepsilon_{\mathrm{c}}(\omega)\left(\varepsilon_{1}-\varepsilon_{\mathrm{ab}}(\omega)\right)}{\varepsilon_{1}^{2}-\varepsilon_{\mathrm{ab}}(\omega) \varepsilon_{\mathrm{c}}(\omega)}}$,

where $\varepsilon_{1}$ is the permittivity of the isotropic medium while $\varepsilon_{\mathrm{ab}}(\omega)$ and $\varepsilon_{c}(\omega)$ are the $a b$-plane and $c$-axis dielectric constants of the anisotropic material. Note that if $\varepsilon_{a b}=\varepsilon_{c}$, we recover the textbook dispersion formula of surface polariton in isotropic media, see ref. ${ }^{9}$ and Supplementary Eq. (7). AFM-SNOM has thus access to both in- and inter-plane charge dynamics, even if just one surface 

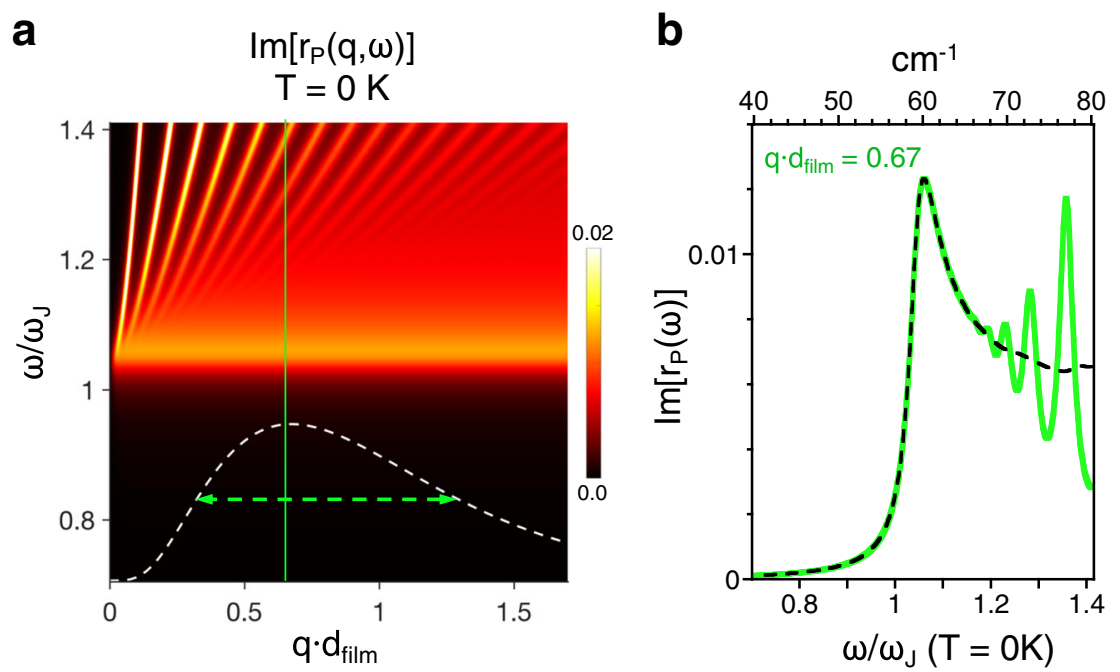

Fig. 3 Dispersions of surface and guided Josephson plasmon modes. a Contour plot of the calculated imaginary part of the Fresnel reflection coefficient $r_{\mathrm{P}}(q, \omega)$ at zero temperature. The vertical axis is in units of $\omega_{\mathrm{J}}$, the screened Josephson plasma frequency. The horizontal axis is in units of $q \cdot d_{\text {film }}$, where $q$ is the momentum and $d_{\text {film }}=13 \mathrm{~nm}$ is the film thickness. The white dashed line is the AFM-SNOM momentum form-factor, see text and Supplementary Note 6 . The green solid line is a constant-momentum cut at the maximum of the distribution (solid line) and the green dashed line marks the full-width at half-maximum (FWHM) of this distribution. $\mathbf{b}$ Constant momentum cuts of $\operatorname{Im}\left[r_{\mathrm{p}}(q, \omega)\right]$ at $T=0 \mathrm{~K}$ from the data in panel (a). The bottom axis is in dimensionless units $\omega / \omega_{\mathrm{J}}$ and the top axis is in units of $\mathrm{cm}^{-1}$. The green solid line corresponds to the momentum cut at the maximum of the form factor. The black dashed line is $\operatorname{Im}\left[r_{\mathrm{p}}(\omega)\right]$ with integrated momentum across the FWHM of the form factor, as indicated by the green dashed line in (a).

is accessible. Note also that far-field techniques require either large single crystals with surfaces parallel to the $c$-axis or, possibly, a grazing-incidence configuration for the case of thin films where the $c$-axis is perpendicular to the surface. Because of deepsubwavelength resolution, crystals or flakes with $\mu \mathrm{m}$-length dimensions are sufficient for AFM-SNOM.

The energies and spectral weights associated with Josephson plasma modes in our film can be seen in Fig. 3, which shows the energy-momentum plot of the Fresnel reflection coefficient $r_{\mathrm{p}}(q$, $\omega)$. The AFM-SNOM momentum form factor is also shown in panel (a) of this figure by a white dashed line. Within the dipole model its functional form is given by $q^{2} \cdot \exp \left[-2 q z_{0}\right]$ where $z_{0}$ is the distance of minimum approach, chosen here to be equal to the tip radius $r_{\text {tip }} \sim 20 \mathrm{~nm}$, see also Supplementary Note 6 . The closely spaced branches above unity represent propagating modes inside the film, while the feature at $\omega / \omega, \sim 1$, nearly dispersionless in this momentum range, correspond to SJPWs. The vertical axis in Fig. 3a represents energy in units of $\omega_{\mathrm{J}}=56.7 \mathrm{~cm}^{-1}$, which is the calculated Josephson plasma frequency for LSCO at $T=0 \mathrm{~K}$. The values of $r_{\mathrm{p}}(q, \omega)$ were calculated using measurements of $c$-axis reflectance in bulk crystals ${ }^{31}$ and in-plane $\mathrm{THz}$ complex conductivity data in single-crystal LSCO films grown by ALL-MBE ${ }^{34}$, see Supplementary Note 2. Our derivation of $r_{\mathrm{P}}(q, \omega)$ generalizes the characteristic matrix formalism for treating anisotropic materials in stratified media, see Supplementary Note 4. This approach also helps generalize Eq. (2) for multilayers because even thin-film devices contain at least two interfaces: vacuumLSCO and LSCO-substrate, in our case. In this configuration there are two SJPW branches whose energies at high momenta can be read off directly from the zeros of the denominator in Eq. (2), inserting for $\varepsilon_{1}$ the values corresponding to vacuum and the substrate, respectively, see also Supplementary Fig. 3. A related approach has been used in ref. ${ }^{49}$ for obtaining surface mode dispersions in topological semiconductors. An important point for our interpretation is the realization that, in spite of the very different physical origin, the near-field signal tracks with a good approximation the screened Josephson plasma frequency. The reason is that the asymptotic values of the SJPWs at high wave- vectors are pushed very close to $\omega_{\mathrm{J}}$ due the strong anisotropy of the ab-plane and c-axis plasma frequencies in LSCO.

Thin films allow for the hybridization of surface modes that are either symmetric or anti-symmetric with respect to the reflection in horizontal symmetry plane. In the presence of a substrate these modes cease to have strict odd/even parity but crosstalk between them is in principle still allowed. In our experiment, i.e. for the range of momenta defined by our form-factor (see white dashed line in Fig. 3a) and energies right below the screened $c$-axis plasma, we are mostly sensitive to the surface mode confined to the top interface. This is because of the large and negative imaginary part of $k_{2 z}$ in Supplementary Eq. (10), the vertical component of the wave momentum inside the slab, which makes $r_{\mathrm{P}}\left(q \approx 1 / d_{\text {film }}, \omega \widetilde{<} \omega_{P, c}\right)$ practically equal to the vacuum-film reflection coefficient $r_{12}$. In other words, the pole in $\operatorname{Im}\left[r_{\mathrm{p}}(q, \omega)\right]$ associated with the mode propagating on the bottom interface has a vanishing strength. Further mathematical and visual explanations are given in Supplementary Notes 4 and 5 and Supplementary Figs. 3, 4.

Furthermore, the energy scale and the momentum distribution of our experiment (the white dashed line in Fig. 3a) also allow us to distinguish between bulk-cavity and surface modes. Propagating Josephson plasmons inside the film correspond to the higher energy branches in Fig. 3a while surface modes appear for our momentum range as a horizontal bright line. Figure $3 \mathrm{~b}$ shows that momentum integration across the full-width-at-half-maximum (FWHM) of the distribution does not affect the surface mode: the green and black dashed lines practically coincide at energies $\omega / \omega_{\mathrm{J}} \sim 1$. However, it flattens out the peak structure of the bulkcavity modes at higher energies. In conclusion, Fig. 3 reveals not only that modes propagating inside the film are at higher energy than the Josephson plasma edge, but also that momentum averaging completely smears out their spectral structure while leaving the surface modes intact because of their dispersionless nature at high momenta. We therefore find that our experiment is sensitive to SJPWs.

Last but not least, the scenario invoking surface Josephson plasmons is also able to explain the temperature dependence 
a

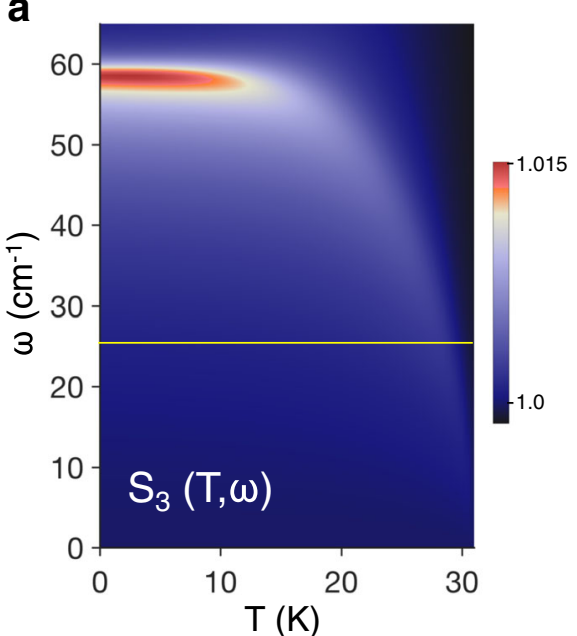

b

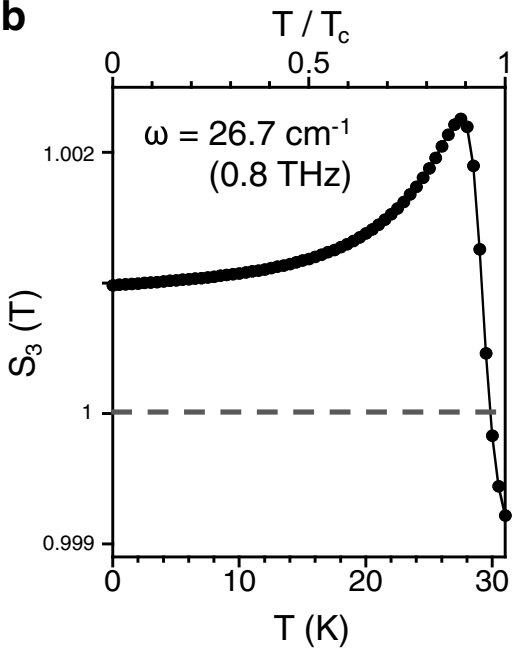

Fig. 4 Temperature and frequency dependence of surface Josephson plasma waves. a A contour plot of the calculated amplitude of the $3^{\text {rd }}$ harmonic near-field signal from LSCO, $S_{3}(T, \omega)$, normalized to Au. The horizontal temperature scale extends from $T=0 \mathrm{~K}$ to the critical superconducting temperature $T_{\mathrm{c}}=31 \mathrm{~K}$. The yellow solid line marks the constant energy cut at $\omega=26.7 \mathrm{~cm}{ }^{-1}(0.8 \mathrm{THz})$. b Temperature dependence of $S_{3}(T)$ for $\omega=26.7 \mathrm{~cm}^{-1}$, the yellow line in panel (a). This is the frequency at which the data in Fig. 2 were acquired. The graydashed line marks equal near-field intensity from LSCO and Au, as in Fig. 2b. Our calculations reproduce well the experimental observations: a non-monotonic temperature dependence of the normalized $S_{3}(T, \omega)$ signal and the reversal of contrast in SNOM signal between LSCO and Au upon entering the superconducting state. This is due to the resonant excitation of SJPWs in the LSCO film.

observed in Fig. 2b. The calculated temperature and frequency dependence of the near-field signal within the spherical-dipole approximation is shown in Fig. 4, see also Supplementary Note 6. In this approximation the entire tip is replaced by a polarizable sphere whose radius is roughly given by the AFM tip apex curvature. The spherical-dipole approach underestimates the experimental contrast and has clear limitations because of its simplifying assumptions. While a quantitative agreement between the model and experiment is not anticipated, we nevertheless expect that the qualitative features of Fig. 2 are captured. Figure 4 shows that this is indeed the case, attesting to the robustness of our interpretation in terms of SJPWs. The increase of the relative LSCO/Au signal above unity in the superconducting state as well as the non-monotonic temperature dependence are naturally understood in this scenario. As shown in Supplementary Fig. 4, in our measurements we change the temperature and use the constant energy of our $\mathrm{THz}$ source. At a given temperature below $T_{c}$, the reflectance edge sweeps across our energy window and that is also the point where the near-field signal is enhanced. With further cooling, the superfluid density increases, the reflectance edge moves to higher energy, leading to a decrease in the nearfield response, which is what we observe experimentally.

\section{DISCUSSION}

As expected, the spherical-dipole model is not able to account quantitatively our data. For reasonable fitting parameters it renders values for the LSCO/Au intensity ratio that are too small. The shortcomings can be understood and are essentially related to the simplifying assumptions about effective tip polarizability, near-field distribution, field enhancement factors and the neglect of retardation effects. Indeed, increasing the tip polarizability or taking into account a more realistic shape of the AFM tip would lead to a larger LSCO/Au contrast than that shown in Fig. 4, see also Supplementary Fig. 5 and the discussion in Supplementary Note 6. Substantial quantitative discrepancies related to the spherical-dipole model were already remarked for experiments performed in the infrared range and more realistic models were proposed for the AFM tip geometry ${ }^{50}$ as well as for the electrodynamic response of the sample-probe system ${ }^{51,52}$. Because in our experiment the wavelengths reach millimeter range, the shortcomings of this model are further exacerbated. Therefore, we believe that a meaningful quantitative agreement requires a full electrodynamical treatment taking accurately into account the shapes of AFM tips and the details of the scanning regime, which is outside the scope of this study.

Using the value of the Josephson plasma frequency $\omega_{\mathrm{J}}=$ $0.8 \mathrm{THz}$ at $T=0.8 T_{\mathrm{c}}$, at optimal doping we obtain a critical current density of the $c$-axis junction $J_{c} \sim 2 \times 10^{5} \mathrm{~A} / \mathrm{cm}^{2}$ and a Josephson penetration depth $\lambda_{\mathrm{J}}=c / \omega_{\mathrm{J}} \sim 375 \mu \mathrm{m}$, indicating that our $20 \mu \mathrm{m}$ wide wire is in a quasi-1D long Josephson junction regime. For insulating barriers, $\omega$, tracks, in turn, the superconducting gap $\Delta$. Using the normal-state conductivity evaluated from far-infrared reflectance data ${ }^{31}$ the gap can be estimated ${ }^{53}$ to $\Delta\left(T=0.8 T_{\mathrm{c}}\right)$ $\sim 24 \mathrm{~cm}^{-1}(0.75 \mathrm{THz})$, see also Supplementary Note 2 . This is a value which is close to the frequency of AFM-SNOM data and it is in very good agreement with recent measurements of the superconducting gap in LSCO-based tunnel junctions ${ }^{54}$. Note that the momentum distribution probed in AFM-SNOM experiments depend on the probe geometry, see Fig. 3a. A systematic exploration of the strong coupling (polaritonic), regime of the SJPW modes is important for probing superconducting nodes or analysis of dissipation channels. This task can be accomplished in several ways. One is by taking advantage of the fact that parameters such as the conical shapes or apex radii can be engineered for etched wire tips ${ }^{55,56}$. Other routes can be provided by 'tilting' the light line dispersion by using thin cover layers of high dielectric permittivity such as $\mathrm{SrTiO}_{3}$, or by using hyperspectral near-field imaging extended to the $\mathrm{THz}$ range ${ }^{57}$. It is important to note that Eq. (2), which deals with dispersion of excitations confined to planar interfaces, has to be amended when the wavelength of these modes is comparable to the sample width, which defines the Sommerfeld or Goubau regime ${ }^{11,58,59}$. A comparison with the momentum selective form-factor of the AFM tip, white dashed line in Fig. 3, shows that in our configuration when the light couples to the sample only through the tip, the contribution of the surface modes in the longwavelength limit is vanishingly small. Consequently, Eq. (2) is 
applicable here for a broad momentum range which includes the relevant contributions for analysis. Note also that the extracted values for the Josephson penetration depth and superconducting gap are not obtained from direct measurements, but rather indirectly from the value of $\omega$, and theoretical considerations in ref. ${ }^{53}$. Measurements of samples with different widths combined with the use of engineered AFM tips with larger apex radii provide a clear path for checking experimentally the role of boundary conditions when exploring the strong light-matter regime.

The energy scales and the temperature dependence of the LSCO/Au near-field ratio constrain the interpretation sufficiently so that we can assign our observations to SJPWs. Other signatures compatible with this interpretation may exist in theory, for example real-space interference patterns of propagating plasmonic excitations ${ }^{8}$. We did not find observe such fringes experimentally. The reasons for their absence can be understood as the result of the $d$-wave gap and strong quasi-particle damping at temperatures $T \approx 0.8 \cdot T_{c}$, which are close to the base temperature of our system. Customized AFM tip radii may be also needed to maximize the coupling efficiency. Plasma waves such as the ones reported here for LSCO have not been observed yet in Fe-based superconductors ${ }^{60}$, although they are layered materials as well. It would therefore be interesting to explore the coupling to these excitations as a function of the sample anisotropy and damping in engineered heterostructures as well as in other cuprate families.

In addition to deep sub-diffractional resolution, light demodulation at different harmonics of the AFM tapping frequency provides dielectric depth information ${ }^{21}$. Thus, cryogenic AFM-SNOM allows probing both the lateral and vertical spatial (in)homogeneity in superconductors, offering a path for mapping superfluid profiles confined to buried interfaces ${ }^{15}$. A variable-temperature near-field environment can also enable characterization of confined modes in topological heterostructures and quantification of length-scales associated to superconducting proximity effects in exposed surfaces or in buried interfaces ${ }^{61}$. Non-linear effects involving Josephson plasma in layered superconductors have been observed in LSCO by far-field methods ${ }^{7,22}$ and they are predicted to be even stronger below $\omega_{\mathrm{J}}$. It is conceivable that electromagnetic field enhancement around the tip will allow further avenues for exploration and control of such photonic effects in the $\mathrm{THz}$ range.

\section{METHODS}

$\mathrm{La}_{1.85} \mathrm{Sr}_{0.15} \mathrm{CuO}_{4}$ (LSCO) film synthesis and device fabrication Optimally doped, LSCO films with a thickness of $13 \mathrm{~nm}$ were grown on LSAO substrates using ALL-MBE. The stoichiometry was controlled before and during deposition by a scanning quartz crystal monitor and by a calibrated custom-built 16-channel atomic absorption spectroscopy system, respectively. Film thickness was monitored in situ by counting the reflection high-energy electron diffraction oscillations and post growth by AFM, profilometry and the finite-thickness oscillations in X-ray reflectance and diffraction. The latter also attest to the film being atomically smooth. The substrate temperature during the growth was $650^{\circ} \mathrm{C}$ and the ozone partial pressure was kept at $p=2 \times 10^{-5}$ Torr. The device, part of which is shown in Fig. $1 \mathrm{~b}$ was patterned using photolithography into a narrow strip with 32 pairs of lateral contact pads, as described in the main text. The data shown in Fig. 2 were acquired from an area in the vicinity of one such lateral contact (marked by a red rectangle in Fig. 1b) and the same results were reproduced for other contacts.

\section{AFM-SNOM measurements}

Near-field and AFM data were acquired simultaneously in a custom-built AFM-SNOM setup. The sample chamber was optimized for laser light access to the AFM tip, sample visualization and a large numerical aperture in the collection path. AFM tips are reproducibly obtained by etching $100 \mu \mathrm{m}$ diameter $\mathrm{W}$ wires in a $2 \mathrm{M}-\mathrm{NaOH}$ solution and using a two-ring geometry. Voltages (peak-to-peak) in the 2-10 V range and frequencies between $5 \mathrm{~Hz}$ and $800 \mathrm{~Hz}$ can be used for shaping the AFM tip shaft and apex radius. The tip radius used here is $\sim 20 \mathrm{~nm}$ as imaged by Scanning Electron Microscopy. The tips were glued to a piezo-actuated quartz tuning fork (TF) resonator operated at its resonance frequency of $28.38 \mathrm{kHz}$ (the resonance frequency without the mounted tip was $2^{15} \mathrm{~Hz}=32.768 \mathrm{kHz}$ ). AFM data were taken in amplitude-modulation mode with the feedback based on the TF piezocurrent. The resonance FWHM was $\sim 5 \mathrm{~Hz}$ and $\sim 1 \mathrm{~Hz}$ at room and low temperatures, respectively. Q-control was used to broaden the TF resonance when the system was cold. The AFM oscillation amplitude was $A \sim 80 \mathrm{~nm}$ as determined from both AFM approach curves as well as from precise interferometric measurements. Light from a Backward Wave Oscillator (Microtech Instruments) with a wavelength $\lambda=375 \mu \mathrm{m}\left(800 \mathrm{GHz}=26.7 \mathrm{~cm}^{-1}\right)$ was focused on the tip with the help of an off-axis Au-coated copper paraboloid. The SNOM signal was focused on a LN2 - LHe cooled hot electron bolometer and demodulated in real-time up to the 4th harmonic of the AFM tapping frequency with the help of a home-made data acquisition system. We analyzed the SNOM data within the dipole model, which roughly approximates the metallic AFM tip with a sphere whose polarizability is modulated by the interaction with the LSCO sample. Details about the model can be found in the Supplementary Note 6.

\section{AFM-SNOM data acquisition (DAQ)}

A custom made DAQ system was built in order to analyze the SNOM signal in-real time, simultaneously with AFM topography data. The hardware consists of a PC computer, a National Instruments high-speed digitizer and a microcontroller. Custom software provides the graphical user interface and synchronizes the operation of the system. The reference clock of the digitizer, reduced to its sampling frequency of a few $\mathrm{MHz}$, is sent to the microcontroller which serves as a digital counter. The microcontroller also triggers the digitizer to start recording time-series data upon receiving pixel pulses from the ASC500 unit, which is the Attocube scanning probe microscope (SPM) controller. At each pulse, the microcontroller sends the current value of its counter to the $P C$ to precisely identify each pixel within the digitizer's data streams. Two data streams are digitized and sent to the PC simultaneously: a reference sine wave output at the tuning fork's resonance frequency $\left(\Omega_{\mathrm{TF}}\right)$ from an output of the ASC500 controller and the actual detector signal. The reference has a dual purpose: (i) it allows for clock-independent measurements of $\Omega_{\mathrm{TF}}$ for subsequent Fourier analysis of the detector signal at each pixel and (ii) it allows the SNOM-DAQ to operate the SPM in both amplitude and frequency modulation modes. A lock-in method allows demodulation of the SNOM signal at desired multiples of $\Omega_{\mathrm{TF}}$. The calculated values for each pixel are then used to construct 2D dielectric SNOM images of the material surface.

\section{DATA AVAILABILITY}

The authors declare that all data supporting the findings of this study are available within the manuscript and the supplementary information. They are available from the corresponding author upon reasonable request.

\section{CODE AVAILABILITY}

All numerical codes in this paper are available upon request to the authors.

Received: 18 April 2020; Accepted: 9 September 2020; Published online: 01 October 2020

\section{REFERENCES}

1. Anderson, P. W. Plasmons, gauge invariance, and mass. Phys. Rev. 130, 439-442 (1963).

2. Carlson, R. V. \& Goldman, A. M. Propagating order-parameter collective modes in superconducting films. Phys. Rev. Lett. 34, 11-15 (1975).

3. Pekker, D. \& Varma, C. M. Amplitude/Higgs modes in condensed matter physics. Annu. Rev. Condens. Matter Phys. 6, 269-297 (2015).

4. Economou, E. N. Surface plasmons in thin films. Phys. Rev. 182, 539-554 (1969).

5. Basov, D. N., Fogler, M. M. \& Garcia de Abajo, F. J. Polaritons in van der Waals materials. Science 354, aag1992 (2016). 
6. Slipchenko, T. M., Kadygrob, D. V., Bogdanis, D., Yampol'skii, V. A. \& Krokhin, A. A. Surface and waveguide Josephson plasma waves in slabs of layered superconductors. Phys. Rev. B 84, 224512 (2011).

7. Laplace, Y. \& Cavalleri, A. Josephson plasmonics in layered superconductors. Adv. Phys.: X 1, 387-411 (2016).

8. Stinson, H. T. et al. Infrared nanospectroscopy and imaging of collective superfluid excitations in anisotropic superconductors. Phys. Rev. B 90, 014502 (2014).

9. Novotny, L. \& Hecht, B. Principles of Nano-Optics. (Cambridge University Press, Cambridge, 2006)

10. Tsiatmas, A. et al. Superconducting plasmonics and extraordinary transmission. Appl. Phys. Lett. 97, 111106 (2010).

11. Schumacher, D., Rea, C., Heitmann, D. \& Scharnberg, K. Surface plasmons and Sommerfeld-Zenneck waves on corrugated surfaces: application to high-T superconductors. Surf. Sci. 408, 203-211 (1998).

12. Dunmore, F. J. et al. Observation of below-gap plasmon excitations in superconducting $\mathrm{YBa}_{2} \mathrm{Cu}_{3} \mathrm{O}_{7}$ films. Phys. Rev. B 52, R731-R734 (1995).

13. Tsiatmas, A., Fedotov, V. A., Garcia de Abajo, F. J. \& Zheludev, N. I. Low-loss terahertz superconducting plasmonics. N. J. Phys. 14, 115006 (2012).

14. Lawrence W. E. \& Doniach S. Proc. 12th Int. Conf. on Low Temperature Physics, Kyoto, Japan (ed Kanda, E.) 361 (Academic Press, 1971).

15. Gozar, A. et al. High-temperature interface superconductivity between metallic and insulating copper oxides. Nature 455, 782-785 (2008).

16. Gozar, A. \& Bozovic, I. High temperature interface superconductivity. Physica $C$ 521, 38-49 (2016).

17. Ritchie, R. H. Plasma losses by fast electrons in thin films. Phys. Rev. 106, 874-881 (1957).

18. Boriskina, S. V. et al. Losses in plasmonics: from mitigating energy dissipation to embracing loss-enabled functionalities. Adv. Opt. Photonics 9, 775-827 (2017).

19. Jia, X. et al. Anomalous acoustic plasmon dispersion from topologically protected states. Phys. Rev. Lett. 119, 136805 (2017).

20. Shvonski, A., Kong, J. \& Kempa, K. Plasmon-polaron of the topological metallic surface states. Phys. Rev. B 99, 125148 (2019).

21. Krutokhvostov, A. A. et al. Enhanced resolution in subsurface near-field optical microscopy. Opt. Express 20, 593-600 (2012)

22. Dienst, A. et al. Optical excitation of Josephson plasma solitons in a cuprate superconductor. Nat. Mater. 12, 535-541 (2013).

23. Savel'ev, S., Yampol'skii, V. A., Rakhmanov, A. L. \& Nori, F. Terahertz Josephson plasma waves in layered superconductors: spectrum, generation, nonlinear and quantum phenomena. Rep. Progr. Phys. 73, 026501 (2010).

24. Savel'ev, S., Rakhmanov, A. L., Yampol'skii, V. A. \& Nori, F. Analogues of nonlinear optics using terahertz Josephson plasma waves in layered superconductors. Nat. Phys. 2, 521-525 (2006).

25. Stern, F. Polarizability of a two-dimensional electron gas. Phys. Rev. Lett. 18 546-548 (1967)

26. Diaconescu, B. et al. Low-energy acoustic plasmons at metal surfaces. Nature $\mathbf{4 4 8}$ 57-59 (2007).

27. Das Sarma, S. \& Quinn, J. J. Collective excitations in semiconductor superlattices. Phys. Rev. B 25, 7603-7618 (1982).

28. Morawitz, H., Bozovic, I., Kresin, V. Z., Rietveld, G. \& van der Marel, D. The plasmon density of states of a layered electron gas. Z. Phys. B 90, 277-281 (1993).

29. Jain, J. K. \& Allen, P. B. Plasmons in layered films. Phys. Rev. Lett. 54, 2437-2440 (1985).

30. Singley, E. J. et al. Measuring the Josephson plasma resonance in $\mathrm{Bi}_{2} \mathrm{Sr}_{2} \mathrm{CaCu}_{2} \mathrm{O}_{8}$ using intense coherent THz synchrotron radiation. Phys. Rev. B 69, 092512 (2004).

31. Tamasaku, K., Nakamura, Y. \& Uchida, S. Charge Dynamics across the $\mathrm{CuO}_{2}$ Planes in $\mathrm{La}_{2-x} \mathrm{Sr}_{x} \mathrm{CuO}_{4}$. Phys. Rev. Lett. 69, 1455-1458 (1992).

32. Basov, D. N., Timusk, T., Dabrowski, B. \& Jorgensen, J. D. c-axis response of $\mathrm{YBa}_{2} \mathrm{Cu}_{4} \mathrm{O}_{8}$ : a pseudogap and possibility of Josephson coupling of $\mathrm{CuO}_{2}$ planes. Phys. Rev. B 50, 3511-3514 (1994).

33. van der Marel, D., Habermaier, H.-U., Heitmann, D., Konig, W. \& Wittlin, A. Infrared study of the superconducting transition in $\mathrm{YBa}_{2} \mathrm{Cu}_{3} \mathrm{O}_{7-x}$. Physica $\mathrm{C}$ (Amsterdam) 176, 1-18 (1991).

34. Bilbro, L. S. et al. Temporal correlations of superconductivity above the transition temperature in $\mathrm{La}_{2-x} \mathrm{Sr}_{x} \mathrm{CuO}_{4}$ probed by terahertz spectroscopy. Nat. Phys. 7 298-302 (2011).

35. Keilmann, F. \& Hillenbrand, R. Near-field microscopy by elastic light scattering from a tip. Philos. Trans. R. Soc. A 362, 787-805 (2004).

36. Atkin, J. M., Berweger, S., Jones, M. B. \& Raschke, M. B. Nano-optical imaging and spectroscopy of order, phases, and domains in complex solids. Adv. Phys. 61, 745-842 (2012).

37. Bozovic, I. Atomic layer engineering of superconducting oxides: yesterday, today, tomorrow. IEEE Trans. Appl. Supercond. 11, 2686-2695 (2001).

38. Gozar, A., Litombe, N. E., Hoffman, J. E. \& Bozovic, I. Optical nanoscopy of high-Tc cuprate nanoconstriction devices patterned by Helium ion beams. Nano Lett. 17, 1582-1586 (2017).
39. Chaplot, S. L., Reichardt, W., Pintschovius, L. \& Pyka, N. Common interatomic potential model for the lattice dynamics of several cuprates. Phys. Rev. B 52, 7230-7242 (1995).

40. Gozar, A., Dennis, B. S., Blumberg, G., Komyia, S. \& Ando, Y. Magnetic order in lightly doped $\mathrm{La}_{2-\mathrm{x}} \mathrm{Sr}_{\mathrm{x}} \mathrm{CuO}_{4}$. Phys. Rev. Lett. 93, 027001 (2004).

41. Dean, M. P. M. et al. Persistence of magnetic excitations in $\mathrm{La}_{2-x} \mathrm{Sr}_{x} \mathrm{CuO}_{4}$ from the undoped insulator to the heavility overdoped non-superconducting metal. Nat. Mater. 12, 1019-1023 (2013).

42. Tajima, S. et al. In-plane optical conductivity of $\mathrm{La}_{2-\mathrm{x}} \mathrm{Sr}_{x} \mathrm{CuO}_{4}$. Reduced superconducting condensate and residual Drude-like response. Phys. Rev. B 71, 094508 (2005).

43. Bergman, D. J. \& Imry, Y. Critical behavior of the complex dielectric constant near the percolation threshold of a heterogeneous material. Phys. Rev. Lett. 39, 1222-1225 (1977).

44. Bonn, D. A., Dosanjh, P., Liang, R. \& Hardy, W. N. Evidence for rapid suppression of quasi-particle scattering below $\mathrm{T}_{\mathrm{c}}$ in $\mathrm{YBa}_{2} \mathrm{Cu}_{3} \mathrm{O}_{7-\delta}$. Phys. Rev. Lett. 68, 2390-2393 (1992).

45. Bardasis, A. \& Schrieffer, J. R. Excitons and plasmons in superconductors. Phys. Rev. 121, 1050-1062 (1961).

46. Sun, Z., Fogler, M. M., Basov, D. N. \& Millis, A. J. Collective modes and THz nearfield response of superconductors. Phys. Rev. Res. 2, 023413 (2020).

47. Artemenko, S. N. \& Kobelkov, A. G. Linear response and collective oscillations in superconductors with $d$-wave pairing. Physica C. 282-287, 1845-1846 (1997).

48. Chen, X. et al. Modern scattering-type scanning near-field optical microscopy for advanced material research. Adv. Mat. 31, 1804774 (2019).

49. Charnukha, A. et al. Ultrafast nonlocal collective dynamics of Kane plasmonpolaritons in a narrow-gap semiconductor. Sci. Adv. 5, eaau9956 (2019).

50. Cvitkovic, A., Ocelic, N. \& Hillenbrand, R. Analytical model for quantitative prediction of material contrasts in scattering-type near-field optical microscopy. Opt Express 15, 8550-8565 (2007).

51. McLeod, A. S. et al. Model for quantitative tip-enhanced spectroscopy and the extraction of nanoscale-resolved optical constants. Phys. Rev. B 90, 085136 (2014).

52. Jiang, B. Y., Zhang, L. M., Castro-Neto, A. H., Basov, D. N. \& Fogler, M. M. Generalized spectral method for near-field optical microscopy. J. Appl. Phys. 119, 054305 (2016).

53. Ambegaokar, V. \& Baratoff, A. Tunneling between superconductors. Phys. Rev. Lett. 10, 486-489 (1963).

54. Zhou, P. et al. Electron pairing in the pseudogap state revealed by shot noise in copper oxide junctions. Nature 572, 493-496 (2019).

55. Ibe, J. P. et al. On the electrochemical etching of tips for scanning tunneling microscopy. J. Vac. Sci. Technol. A 8, 3570-3575 (1990).

56. Borzenets, I. V. et al. Ultra-sharp metal and nanotube-based probes for applications in scanning microscopy and neural recording. J. Appl. Phys. 111, 074703 (2012).

57. Sterczewski, L. A. et al. Terahertz hyperspectral imaging with dual chip-scale combs. Optica 6, 766-771 (2019).

58. Goubau, G. Surface waves and their application to transmission lines. J. Appl. Phys. 21, 1119-1128 (1950).

59. Sergeichev, K. F., Karfidov, D. M., Andreeev, S. E., Sizov, Y. E. \& Zhukov, V. I. Excitation and propagation of Sommerfeld-Zenneck surface waves on a conducting strip in the centimeter-wave band. J. Commun. Technol. Electron. 63 326-334 (2018).

60. Zhang, S. et al. Lattice dynamics of ultrathin FeSe films on $\mathrm{SrTiO}_{3}$. Phys. Rev. Lett. 97, 035408 (2018).

61. Woods, B. D., Das Sarma, S. \& Stanescu, T. D. Electronic structure of full-shell InAs/ Al hybrid semiconductor-superconductor nanowires: spin-orbit coupling and topological phase space. Phys. Rev. B 99, 161118(R) (2019).

\section{ACKNOWLEDGEMENTS}

A.G. and X.H. were supported by the Gordon and Betty Moore Foundation's EPiQS Initiative through Grant GBMF4410. A.T.B and I.B. were supported by the U.S. Department of Energy, Basic Energy Sciences, Materials Sciences and Engineering Division. A. G. also acknowledges support from the DOE Early Career Research program (Grant 2005410). Q.L and A.G. acknowledge support from the Yale West Campus Materials Characterization Core for SEM imaging of AFM tips.

\section{AUTHOR CONTRIBUTIONS}

Q.L. and A.G. took and analyzed the SNOM-AFM data. The synthesis of LSCO films by ALL-MBE and characterization were done at Brookhaven by X.H., A.T.B., and I.B. Lithography and Hall bar characterization by transport was done by A.T.B. R.S., and A. G. designed and built the AFM-SNOM data acquisition system. A.G. led the experiment and wrote the manuscript with contribution from all authors. 


\section{COMPETING INTERESTS}

The authors declare no competing interests.

\section{ADDITIONAL INFORMATION}

Supplementary information is available for this paper at https://doi.org/10.1038/ s41535-020-00272-8.

Correspondence and requests for materials should be addressed to A.G.

Reprints and permission information is available at http://www.nature.com/ reprints

Publisher's note Springer Nature remains neutral with regard to jurisdictional claims in published maps and institutional affiliations.
Open Access This article is licensed under a Creative Commons Attribution 4.0 International License, which permits use, sharing, adaptation, distribution and reproduction in any medium or format, as long as you give appropriate credit to the original author(s) and the source, provide a link to the Creative Commons license, and indicate if changes were made. The images or other third party material in this article are included in the article's Creative Commons license, unless indicated otherwise in a credit line to the material. If material is not included in the article's Creative Commons license and your intended use is not permitted by statutory regulation or exceeds the permitted use, you will need to obtain permission directly from the copyright holder. To view a copy of this license, visit http://creativecommons. org/licenses/by/4.0/.

(c) The Author(s) 2020 